\title{
A Complication-Based Comparison Between the Posterior and Direct Lateral Approaches to Total Hip Arthroplasty: A Single-Center Experience
}

Wazzan ALJuhani ${ }^{1}$, Khalid Alshuwaier $^{2}$, Fisal Alkhamis ${ }^{2}$, Mohammed Q. Alosaimi ${ }^{2}$, Abdullah Alaidroos ${ }^{2}$ , Mohammad A. Alghafees ${ }^{2}$, Emad Masuadi ${ }^{3}$

1. Surgery, King Abdulaziz Medical City, Riyadh, SAU 2. Medicine, King Saud Bin Abdulaziz University for Health Sciences, Riyadh, SAU 3. Research Unit/Biostatistics, King Saud Bin Abdulaziz University for Health Sciences, Riyadh, SAU

Corresponding author: Mohammad A. Alghafees, alghafees687@gmail.com

\section{Abstract \\ Introduction}

Many approaches to performing total hip arthroplasty (THA) exist, primarily due to an insufficient amount of research that would favor one approach over the other. This study aimed to compare the risk of nerve injury, dislocation, Trendelenburg gait, and stem malposition between the direct lateral and posterior approaches to THA.

\section{Methods}

The study was a retrospective cohort study, and it was conducted in King Abdulaziz Medical City. It was directed toward adult patients who underwent THA from November 1, 2003, to November 1, 2018. All figures were obtained through the BESTCare system (ezCareTech, Saudi Arabia). Categorical variables were presented as frequencies and proportions. Quantitative variables were measured as mean and standard deviation. Fisher's exact test was used to compare the risk of complications between the two approaches.

\section{Results}

The posterior approach displayed a greater risk of stem malposition $(\mathrm{p}<0.001)$ when compared with the direct lateral approach. However, neither approach showed a higher risk of dislocation, nerve injury, or Trendelenburg gait.

\section{Conclusions}

A higher risk of stem malposition was observed in the posterior approach, and there were no remarkable variances between the two approaches in the risk of dislocation, nerve injury, and Trendelenburg gait. Until more meticulous nationwide studies are available to provide evidence that would favor an approach over the other, the choice of surgical approach for THA remains to depend on the patient's characteristics and the surgeon's prior experience. Regardless of the approach, preoperative, intraoperative, and postoperative factors that increase the risk of complications should also be identified and addressed adequately.

Categories: Orthopedics

Keywords: arthroplasty, complications, orthopaedic surgery, surgical approaches, saudi arabia

\section{Introduction}

Total hip arthroplasty (THA) is one of the most common procedures in the world. For instance, in 2010, total hip replacement occurrence in the United States was less than $1 \%$ of all arthroplasty surgeries. The estimation was 2.5 million individuals divided into 1.4 million females and 1.1 million males [1].

There is a plethora of approaches to performing total hip arthroplasty. A surgeon can use any approach depending on their own personal preference; because, regarding safety, no adequate amount of convincing research would favor an approach over the other. The posterior and direct lateral approaches are currently two of the most widely used approaches [2].

This study aimed to compare the risk of nerve injury, dislocation, Trendelenburg gait, and stem malposition between both the direct lateral and posterior approaches to the THA procedure in King Abdulaziz Medical City. This research has substantial significance due to the lack of similar studies in the region, i.e., the Middle East.

\section{Materials And Methods}


The study design is a retrospective cohort and conducted at King Abdulaziz Medical City, Riyadh, Saudi Arabia (KAMC). Were incorporated in the study, adult patients who underwent THA in the posterior or direct lateral approaches at KAMC with full medical records from November 1, 2003, to November 1, 2018. Patients who underwent THA outside of KAMC but were being followed up in the aforementioned hospital or underwent THA in an approach other than the posterior or direct lateral were excluded from the study.

The BESTCare system (ezCareTech, Saudi Arabia) was used to collect data. The study included all adult patients who undertook THA in the specified time period except those who have not matched the inclusion criteria. The main resulted variables were nerve injury, dislocation, Trendelenburg gait, and stem malposition. Patients' information was used to group the variables, including age, gender, body mass index (BMI), comorbidities, indications, and admission type.

Figures were conducted by Microsoft Excel 2019 (Microsoft Corporation, WA, USA) and were studied using the Statistical Package for the Social Sciences (SPSS) version 23.0 (IBM Corporation, NY, USA). Frequencies and proportions were brought about for categorical variables, whereas mean and standard deviation were computed for numerical variables. To make a comparison of the risk of complications between the two approaches, Fisher's exact test was used. A p-value of $\leqslant 0.05$ was deemed significant.

The Institutional Review Board of King Abdullah International Medical Research Center, the Ministry of National Guard Health Affairs, Riyadh, Kingdom of Saudi Arabia, approved the present study with approval number SP19/369/R. Patients' confidentiality was ensured. Only the research team members used and collected the data and serial numbers were used instead of medical record numbers.

\section{Results}

Of the 126 participants, $64.3 \%(n=81)$ were males and $35.7 \%(n=45)$ were females. The mean age was 49.32 \pm 19.56 years. The mean body mass index (BMI) was $29.14 \pm 6.44 \mathrm{~kg} / \mathrm{m} 2$, as shown in Table 1 . 


\section{Cureus}

Demographical Data

Age (Mean, SD)

Male (N, \%)

Female (N, \%)

35.70

Body Mass Index (Mean, SD)

Comorbidities

Sickle Cell Disease

29.14

Dyslipidemia

Hypertension

\section{Diabetes Mellitus}

Hypothyroidism

Hip Dysplasia

Hepatitis C

$$
8
$$$$
6.30
$$

6.30

6

\section{Renal Failure}

Osteoporosis

Deep Vein Thrombosis

Benign Prostatic Hyperplasia

Congestive Heart Failure

Arrhythmias

Chronic Obstructive Pulmonary Disease

Rheumatoid Arthritis

Spondylitis

Osteoarthritis

TABLE 1: The Demographic Profile and Comorbidities of The Study Participants ( $N=126)$

Of the comorbidities, the most common was hypertension (32.5\% [n $=41])$ followed by dyslipidaemia $(20.6 \%$ $[\mathrm{n}=26])$, diabetes mellitus $(13.5 \%[\mathrm{n}=17])$, and congestive heart failure $(9.5 \%[\mathrm{n}=12])$, as shown in Table 1 .

Of the 126 participants, 77\% ( $\mathrm{n}=97)$ were admitted for THA electively, and 23\% ( $\mathrm{n}=29)$ were admitted urgently. Among the different indications, avascular necrosis was the most common $(36.5 \%[n=46])$ followed by post-traumatic osteoarthritis $(19.8 \%[\mathrm{n}=25])$, arthritis $(17.5 \%[\mathrm{n}=22])$ and fractures $(13.5 \%[\mathrm{n}=$ 17]). The direct lateral approach of THA was done on $86.5 \%(n=109)$ while $13.5 \%(n=17)$ underwent THA in the posterior approach. The most common surgical complication observed was dislocation ( $11.9 \%[n=15])$, followed by nerve injury $(8.7 \%[n=11])$, stem malposition $(6.3 \%[n=8])$, and Trendelenburg gait $(6.3 \%[n=$ 8]), as shown in Table 2. 


\section{Cureus}

\begin{tabular}{|c|c|c|}
\hline Admission Type & $\mathbf{N}$ & $\%$ \\
\hline Elective & 97 & 77 \\
\hline Urgent & 29 & 23 \\
\hline Indication & $\mathrm{N}$ & $\%$ \\
\hline Avascular Necrosis & 46 & 36.5 \\
\hline Fractures & 17 & 13.5 \\
\hline Rheumatic Diseases & 1 & 0.8 \\
\hline Arthritis & 22 & 17.5 \\
\hline Post-traumatic Osteoarthritis & 25 & 19.8 \\
\hline Dysplasia & 15 & 11.9 \\
\hline Surgical Approach & $\mathrm{N}$ & $\%$ \\
\hline Posterior Approach & 17 & 13.50 \\
\hline Direct Lateral Approach & 109 & 86.5 \\
\hline Complication & $\mathrm{N}$ & $\%$ \\
\hline Trendelenburg Gait & 8 & 6.3 \\
\hline Nerve Injury & 11 & 8.7 \\
\hline Dislocation & 15 & 11.9 \\
\hline Stem Malposition & 8 & 6.3 \\
\hline
\end{tabular}

TABLE 2: Operation Details and Complications $(\mathrm{N}=126)$

A substantial difference was detected between the two approaches regarding having stem malposition, where a higher risk of stem malposition was observed in the posterior approach $(p<0.001)$. No significant differences were observed between the two approaches as per the risks of dislocation, nerve injury, and Trendelenburg gait, as shown in Table 3. 


\section{Cureus}

\begin{tabular}{|c|c|c|c|c|c|}
\hline \multirow{3}{*}{$\begin{array}{l}\text { Complication } \\
\text { Trendelenburg Gait }\end{array}$} & \multicolumn{4}{|c|}{ Type of Surgical Approach } & \multirow{2}{*}{ P-Value } \\
\hline & \multicolumn{2}{|c|}{ Posterior Approach ( $\mathrm{N}=17)$} & \multicolumn{2}{|c|}{ Direct Lateral Approach $(\mathrm{N}=109)$} & \\
\hline & $\mathrm{N}$ & $\%$ & $\mathrm{~N}$ & $\%$ & \multirow{3}{*}{0.248} \\
\hline Yes & 0 & 0.00 & 8 & 7.34 & \\
\hline No & 17 & 100.00 & 101 & 92.66 & \\
\hline Nerve Injury & $\mathrm{N}$ & $\%$ & $\mathrm{~N}$ & $\%$ & \multirow{3}{*}{0.655} \\
\hline Yes & 1 & 94.10 & 10 & 90.80 & \\
\hline No & 16 & 5.90 & 99 & 9.20 & \\
\hline Dislocation & $\mathrm{N}$ & $\%$ & $\mathrm{~N}$ & $\%$ & \multirow{3}{*}{0.103} \\
\hline Yes & 0 & 0.00 & 15 & 13.80 & \\
\hline No & 17 & 100.00 & 94 & 86.20 & \\
\hline Stem Malposition & $\mathrm{N}$ & $\%$ & $\mathrm{~N}$ & $\%$ & \multirow{3}{*}{$<0.001^{*}$} \\
\hline Yes & 7 & 41.20 & 1 & 0.90 & \\
\hline No & 10 & 58.80 & 108 & 99.10 & \\
\hline
\end{tabular}

\section{TABLE 3: Surgical Approach-Based Comparison of Complications}

\section{Discussion}

The study aimed at comparing the risks of nerve injury, dislocation, Trendelenburg gait, and stem malposition between the direct lateral and posterior approaches to total hip arthroplasty in KAMC. We observed a higher risk of stem malposition in the posterior approach than the direct lateral approach. However, the study found no principal variances between the two approaches as per the risks of dislocation, nerve injury, and Trendelenburg gait.

The respective superiority of a THA approach over the other is a controversial subject, with conflicting published results. Unlike a British meta-analysis, which found the posterior approach to have a lower risk of stem malposition, the present study found that the posterior approach had a higher chance of stem malposition than the lateral approach. The study also found the posterior approach to have a lower risk of Trendelenburg gait, which also contradicts our findings. However, the study did not find any difference regarding dislocation risk between the two approaches, which is consistent with the presented findings [3]. Moreover, unlike a systemic review that found a higher risk of nerve injury in the direct lateral approach, our study found no significant difference between the two approaches. However, akin to our negative findings, the same study found that none of the two approaches showed a higher risk of dislocation and postoperative Trendelenburg gait [4]. Moreover, dissimilar to a Swedish study that uncovered a larger chance of dislocation when using the posterior approach, the current study found no difference in dislocation peril between the posterior and direct lateral approaches [5].

Furthermore, a prospective American study found no statistically significant difference between the posterior and lateral approaches in nerve palsy risk, which complements our study's negative findings [6]. On the contrary, a high-potential Brazilian study found an association between using the direct lateral approach and the risk of damage to the superior gluteal nerve, which contradicts our findings [7]. A metaanalysis assessing the risk of dislocation in each of the three most common approaches, including the posterior and the direct lateral approach, found that the dislocation rates are similar, further complementing our negative findings [8]. Furthermore, an additional meta-analysis did not find any association between a greater peril of dislocation and the posterior approach [9]. Inversely, a paper reviewing 260 clinical studies specified that from the data collected, the dislocation rate following THA was 5.9 times higher with the posterior approach compared to the direct lateral approach, which in turn conflicts with our negative findings [10]. Also, a Swiss case-control study found that patients undergoing the posterior approach were six times more prone to experiencing dislocation than the direct lateral approach [11]. In contrast, a South Korean study found that patients are less prone to developing a dislocation when undergoing THA in the posterior approach than in the lateral approach [12]. Moreover, a Dutch study found that following governing relevant perplexing variables, the lateral approach to THA presented a higher risk of stem malposition when compared with the posterior approach, which contradicts our findings [13]. 
Regardless of the approach, these complications can be prevented through preoperative and intraoperative measures. According to a Spanish cohort study, the capsular repair and transosseous reattachment of the external rotators can excellently decrease the peril of dislocation in patients undergoing THA in the posterior approach [14]. Also, a French multicentre case-control study found that an unrepaired joint capsule is considered a significant risk factor for dislocation in patients undergoing THA in any approach. However, the risk was highest in the group that underwent THA in the posterior approach [15]. Also, a British study found that age extremes, a BMI $>30 \mathrm{~kg} / \mathrm{m}^{2}$, lumbosacral disease, and femoral head size can all influence the rates of dislocation post THA irrespective of the approach used [16]. Furthermore, a German study stated that the observed critical trochanter angle in preoperative planning could help in predicting the risk for stem malpositioning. The study recommended that an intraoperative C-ray be used to verify the correct positioning of an implant in patients with a critical trochanteric angle under $20^{\circ}$ or above $30^{\circ}$ [17].

There were some limitations to the study. First, the population size was relatively smaller than global, multicentre studies. Second, due to the study's retrospective nature, the data in the BESTCare system might not be complete. Concerning strengths, first, the current study is the first of its kind in the Middle East and would be a good initiative for conducting more extensive studies that include nationwide databases. Second, King Abdulaziz Medical City follows similar prehabilitation and rehabilitation protocols for all patients undergoing THA, which further solidifies the evidence of approach-related complications.

\section{Conclusions}

A higher risk of stem malposition was observed in the posterior approach, and no significant differences were found between the two approaches in the risk of dislocation, nerve injury, and Trendelenburg gait. Until more meticulous nationwide studies are available to provide strong evidence that would favor one approach over the other, the choice of surgical approach for THA remains dependent on the patient's characteristics and the surgeon's experience. Due to the lack of studies, we recommend that more clinical trials and more extensive nationwide database cohort studies be conducted to explore more factors that would give an advantage to one approach over the other. Future research should also focus on the long-term impact of different surgical approaches on the patient's prognosis. Regardless of the approach, the preoperative, intraoperative, and postoperative factors that increase the risk of complications should also be identified and addressed.

\section{Additional Information \\ Disclosures}

Human subjects: Consent was obtained by all participants in this study. King Abdullah International Medical Research Center issued approval SP19/369/R. Animal subjects: All authors have confirmed that this study did not involve animal subjects or tissue. Conflicts of interest: In compliance with the ICMJE uniform disclosure form, all authors declare the following: Payment/services info: All authors have declared that no financial support was received from any organization for the submitted work. Financial relationships: All authors have declared that they have no financial relationships at present or within the previous three years with any organizations that might have an interest in the submitted work. Other relationships: All authors have declared that there are no other relationships or activities that could appear to have influenced the submitted work.

\section{References}

1. Kremers HM, Larson DR, Crowson CS, et al.: Prevalence of total hip and knee replacement in the united states. J Bone Joint Surg Am. 2015, 97:1386-1397. 10.2106/jbjs.n.01141

2. Post Z, Moretti V: Surgical approaches for total hip arthroplasty. Indian J Orthop. 2017, 51:368-376.

3. Berstock J, Blom A, Beswick A: A systematic review and meta-analysis of complications following the posterior and lateral surgical approaches to total hip arthroplasty. Ann R Coll Surg Engl. 2015, 97:11-16. 10.1308/003588414x13946184904008

4. Jolles B, Bogoch E: Posterior versus lateral surgical approach for total hip arthroplasty in adults with osteoarthritis. Cochrane Database Syst Rev. 2003, 2003:CD003828. 10.1002/14651858.cd003828.pub2

5. Skoogh O, Tsikandylakis G, Mohaddes M, et al.: Contemporary posterior surgical approach in total hip replacement: still more reoperations due to dislocation compared with direct lateral approach? An observational study of the Swedish Hip Arthroplasty Register including 156,979 hips. Acta Orthop. 2019, 90:411-416. 10.1080/17453674.2019.1610269

6. Navarro R, Schmalzried T, Amstutz H, Dorey F: Surgical approach and nerve palsy in total hip arthroplasty. J Arthroplasty. 1995, 10:1-5.

7. Picado CHF, Garcia FL, Marques W: Damage to the superior gluteal nerve after direct lateral approach to the hip. Clin Orthop Relat Res. 2007, 455:209-211. 10.1097/01.blo.0000238805.87411.e8

8. Kwon MS, Kuskowski M, Mulhall KJ, Macaulay W, Brown TE, Saleh KJ: Does surgical approach affect total hip arthroplasty dislocation rates?. Clin Orthop Relat Res. 2006, 447:34-38. 10.1097/01.blo.0000218746.84494.df

9. Miller LE, Gondusky JS, Bhattacharyya S, Kamath AF, Boettner F, Wright J: Does surgical approach affect outcomes in total hip arthroplasty through 90 days of follow-up? A systematic review with meta-analysis. J Arthroplasty. 2018, 33:1296-1302. 10.1016/j.arth.2017.11.011

10. Masonis JL, Bourne RB: Surgical approach, abductor function, and total hip arthroplasty dislocation. Clin Orthop Relat Res. 2002, 405:46-53. 10.1097/00003086-200212000-00006 


\section{Cureus}

11. Dudda M, Gueleryuez A, Gautier E, Busato A, Roeder C: Risk factors for early dislocation after total hip arthroplasty: a matched case-control study. J Orthop Surg. 2010, 18:179-183. 10.1177/230949901001800209

12. Ji H-M, Kim K-C, Lee Y-K, Ha Y-C, Koo K-H: Dislocation after total hip arthroplasty: a randomized clinical trial of a posterior approach and a modified lateral approach. J Arthroplasty. 2012, 27:378-385.

10.1016/j.arth.2011.06.007

13. Janssen L, Wijnands KAP, Janssen D, Janssen MWHE, Morrenhof JW: Do stem design and surgical approach influence early aseptic loosening in cementless THA?. Clin Orthop Relat Res. 2018, 476:1212-1220. 10.1007/s11999.0000000000000208

14. Hernández A, Nuñez J, Mimendia I, Barro V, Azorin L: Early dislocation in primary total hip arthroplasty using a posterior approach with repair of capsule and external rotators. Rev Esp Cir Ortop Traumatol. 2018 62:421-427. 10.1016/j.recote.2018.11.001

15. Fessy M, Putman S, Viste A, et al.: What are the risk factors for dislocation in primary total hip arthroplasty? A multicenter case-control study of 128 unstable and 438 stable hips. Orthop Traumatol Surg Res. 2017, 103:663-668. 10.1016/j.otsr.2017.05.014

16. Rowan FE, Benjamin B, Pietrak JR, Haddad FS: Prevention of dislocation after total hip arthroplasty . J Arthroplasty. 2018, 33:1316-1324. 10.1016/j.arth.2018.01.047

17. Haversath M, Busch A, Jäger M, Tassemeier T, Brandenburger D, Serong S: The 'critical trochanter angle': a predictor for stem alignment in total hip arthroplasty. J Orthop Surg Res. 2019, 14:165. 10.1186/s13018019-1206-x 